\title{
Performance of a Prototype CMS Hadron Barrel Calorimeter in a Test Beam ${ }^{1}$
}

\author{
Arie Bodek \\ Department of Physics \& Astronomy \\ University of Rochester \\ Rochester, NY 14627, USA
}

\begin{abstract}
We report on the performance of a prototype CMS Hadron Barrel Calorimeter (HCAL) module in a test beam. The prototype sampling calorimeter used copper absorber plates and scintillator tiles with wavelength shifting fibers for readout. Placing a lead tungstate crystal electromagnetic calorimeter in front of HCAL affects the linearity and energy resolution of the combined system to hadrons. The data are used to optimize the choice of total absorber depth, sampling frequency, and longitudinal readout segmentation.
\end{abstract}

\section{INTRODUCTION}

We report on a variety of tests performed in a test beam with a prototype CMS Hadron Barrel Calorimeter [1], 2] (HCAL) module in 1995-96. The prototype sampling calorimeter is constructed from copper absorber plates and scintillator tiles with wave length shifter (WLS) fibers for readout [3]. Data were taken both with and without a prototype lead tungstate electromagnetic calorimeter (ECAL) placed upstream of the HCAL module.

In this report, we discuss the performance of HCAL, including the effects of the lead tungstate crystals on the linearity and energy resolution of the response of the combined ECAL+HCAL system to hadrons. This study is used in the optimization of the HCAL design, including the choice of total absorber depth, sampling frequency, and longitudinal readout segmentation.

The CMS Central HCAL calorimeter will operate inside a 4 Tesla magnetic field. A measurement of the effects of a magnetic field on the response of HCAL to muons, electrons and pions is presented elsewhere. [ [

\section{EXPERIMENTAL SETUP}

The CMS combined calorimetric ECAL+HCAL system was tested in 1995 and 1996 at the H2 and H4 CERN beamlines. Data were taken using beams of muons, electrons and hadrons, ranging in momenta from 15 to $375 \mathrm{GeV} / \mathrm{c}$. Test beam prototypes of HCAL were based on the hanging file structure [5]. Copper $\left(\lambda_{I N T}(\mathrm{Cu})=15.06 \mathrm{~cm}\right)$ absorber plates varying in thickness from $2 \mathrm{~cm}$ to $10 \mathrm{~cm}$ were interspersed with scintillator tiles read out with WLS fibers. The total interaction depth of HCAL prototypes corresponds to $8.5 \lambda_{I N T}(\mathrm{H} 4,1995$ module) and $10 \lambda_{I N T}(\mathrm{H} 2,1996$ module). The transverse size of the calorimeter is $64 \mathrm{~cm} \times 64 \mathrm{~cm}$. Each scintillator layer is

\footnotetext{
${ }^{1}$ Representing the CMS HCAL Group at the IEEE Conference, Toronto, Nov. 1998 (Univ. of Rochester Preprint UR-1544 ER/40685/923).
}

read out independently by conventional photomultiplier tubes (PMT). The light yield of each scintillator layer corresponds to approximately to 2 photoelectrons per minimum ionizing particle (2 PE/mip). The relative calibration of scintillator layers is established to accuracy of $\approx 3 \%$ by equalizing the average response of each layer to muons. The average pulse height deposited by muons in HCAL corresponds to approximately $4 \mathrm{GeV}$ (equivalent hadronic energy).

The prototype ECAL detector consists of a matrix of $7 \times 7 \mathrm{PbWO}_{4}$ crystals, $23 \mathrm{~cm}$ long $\left(25.8 \mathrm{X}_{0}, 1.1 \lambda_{I N T}\right)$, each $2 \mathrm{~cm} \times 2 \mathrm{~cm}$ in transverse size. The calibration of the ECAL crystals is done using $50 \mathrm{GeV} / \mathrm{c}$ electrons directed into center of each crystal. The electronic noise of the $7 \times 7$ crystal matrix energy sum had a RMS width equivalent to $440 \mathrm{MeV}$ (much better performance is expected in future prototypes). The absolute energy calibration of HCAL is set using $50 \mathrm{GeV} / \mathrm{c}$ pions interacting in HCAL only. The absolute energy scale of ECAL is set using $50 \mathrm{GeV} / \mathrm{c}$ electrons.

\section{THE $e / h$ RESPONSE RATIO OF HCAL}

The performance of a hadron calorimeter, i.e. hadron energy response linearity and energy resolution, depends [6] on the intrinsic $e / h$, the ratio of the response to the electromagnetic and hadronic shower components. The average fraction of the electromagnetic component, $\mathrm{F}\left(\pi^{0}\right)$ in pion induced showers increases as a function of incident energy. For non-compensating calorimeters $(e / h \neq 1)$, this implies a non-linear hadron energy response. The event-by-event fluctuations in $\mathrm{F}\left(\pi^{0}\right)$ contribute to variations in the reconstructed shower energy and at high energies dominate the energy resolution for hadrons.

We have measured the linearity of the energy response of HCAL to pions which did not interact in ECAL. There is approximately a 9\% increase in the response between 15 and $375 \mathrm{GeV} / \mathrm{c}$. In a separate test, the response of HCAL to electrons was measured by moving the ECAL module out of the beamline. The response of the HCAL to electrons is linear within $2 \%$ and approximately $20 \%$ higher than the average response to pions.

Using these two data sets (the response of HCAL to electrons and response to pions which interacting in HCAL only) we extract the value of the intrinsic $e / h$ of HCAL. Two available parameterizations for $\mathrm{F}\left(\pi^{0}\right)$, one by Wigmans [6] and the other by Groom [7], yield values of $e / h$ of $1.38 \pm$ 0.01 and $1.49 \pm 0.01$, respectively ( see Fig. 1). The simple Wigmans formula for $\mathrm{F}\left(\pi^{0}\right)$ increases with energy $(\approx \log (\mathrm{E}))$ and becomes non-physical at very high energies. The Groom's 
parameterization of GCALOR Monte Carlo simulations uses an exponential form.

\section{LINEARITY AND ENERGY RESOLUTION OF THE $\mathrm{PBWO}_{4}$ ECAL+HCAL}

Figure 2 and 3 show comparisons of the energy response and relative energy resolutions of the combined $\mathrm{PbWO}_{4}$ ECAL+HCAL calorimeters to pions. The inclusive set of pions which interact in either ECAL or HCAL have approximately $10 \%$ lower response than the response of those pions which interact in HCAL only. In addition, the relative energy resolution of the combined calorimetric system is significantly degraded, as compared to the energy resolution of the standalone HCAL system. We attribute these results to the large $e / h$ of the $\mathrm{PbWO}_{4}$ ECAL system.

We have tested two possible approaches to correct for the degradation of the performance of the combined ECAL+HCAL calorimeters. Both of these methods make use of the segmented readout of the HCAL. The first readout segment $(\mathrm{H} 1)$ covers a sampling immediately downstream of the ECAL. The second readout segment $(\mathrm{H} 2)$ includes the remainder of HCAL inside the magnet coil. A third readout segment $(\mathrm{HO})$ corresponds to the HCAL sampling located outside of the magnet cryostat.

In the first approach, called passive weighting, we reduce the non-linearity of energy response $(E / p)$ and relative energy resolution $(\mathrm{rms}(\mathrm{E}) / \mathrm{E})$ by increasing the weight $(\alpha)$ of the first (H1) HCAL readout segment, where $\alpha$ is energy independent constant.

$$
E_{T O T}=E_{E C A L}+\alpha \times E_{H 1}+E_{H 2}+E_{H O}
$$

In the second approach we use a dynamic correction to reduce the effect of the large $e / h$ value of the $\mathrm{PbWO}_{4}$ ECAL calorimeter. This event by event correction, which depends on the fraction of the energy deposited in the first readout segment of HCAL immediately downstream of the ECAL effectively allows one to have a larger correction for the low ECAL response to pions interacting in ECAL.

$$
\begin{gathered}
E_{T O T}=(1+2 \times f(H 1)) \times E_{E C A L}+E_{H 1}+E_{H 2}+E_{H O},(2) \\
f(H 1)=E(H 1) /(E(H 1)+E(H 2)+E(H O)), f(H 1) \leq 0.1
\end{gathered}
$$

Using either the passive or dynamic correction technique, one finds a residual energy nonlinearity of only $10 \%$ for pions with energy between 30 and $300 \mathrm{GeV} / \mathrm{c}$. Note that while the passive weighting method can be applied to single particles as well as to the multiparticle events (jets), the dynamic weighting method may result in high energy tails for $\pi^{0} \pi^{ \pm}$multiparticle jets. If one uses the passive weighting scheme, then a separate readout for $\mathrm{H} 1$ is not needed.

\section{Optimization OF THE HCAL DESIGN}

During the H2(1996) tests, the prototype HCAL module was segmented longitudinally into 27 readout layers. Using the test beam data, we have simulated various sampling configurations in order to study the performance of HCAL as a function of the total interaction length and sampling frequency. The average longitudinal profiles of pions extend past the magnetic coil. This motivated the decision to instrument the CMS muon system iron absorbers (located outside the coil) with scintillator plates for use as a pion shower tail catchers. We refer to this part of the calorimeter as the HCAL Outer (HO) system. By adding the HO, the gaussian parts, as well as the non-gaussian low energy tails in the energy distributions are significantly reduced.

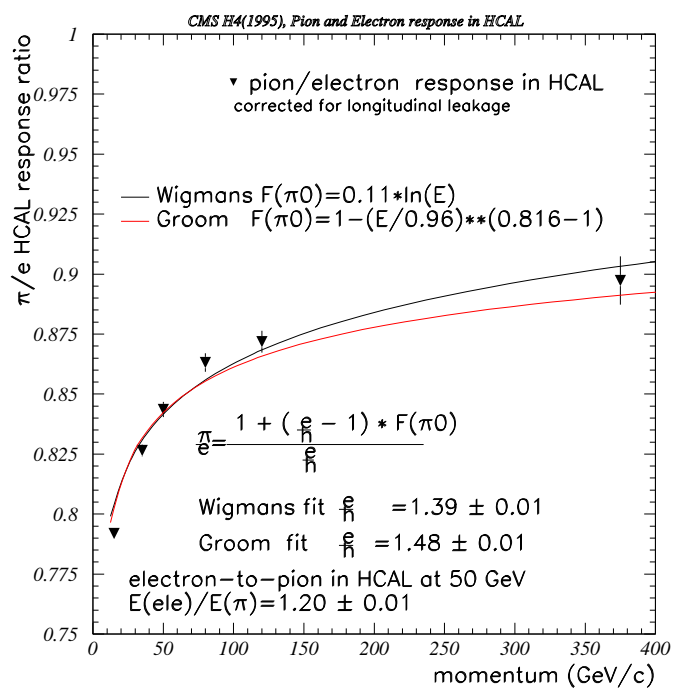

Figure 1: H4(1995) data: the pion/electron ratio of response of the copper sampling prototype HCAL as a function of beam momentum. The calorimeter consists of the Inner HCAL (ten $3 \mathrm{~cm} \mathrm{Cu}$ samplings followed by nine $6 \mathrm{~cm} \mathrm{Cu}$ samplings), the magnetic coil mimic $(8 \mathrm{~cm}$ $\mathrm{Cu}+29 \mathrm{~cm} \mathrm{Al}$ ) and the HCAL Outer (two $8 \mathrm{~cm} \mathrm{Cu}$ and two $10 \mathrm{~cm}$ $\mathrm{Cu}$ samplings). The scintillator is $4 \mathrm{~mm}$ thick SCSN-81. The pion response of HCAL has been corrected for longitudinal leakage. We have assumed a linear electron response of $\operatorname{HCAL}, \mathrm{E}(\mathrm{ele}) / \mathrm{E}(\pi$ at 50 $\mathrm{GeV})=1.20 \pm 0.01$. The extracted values of $e / h$ correspond to the two different parameterizations of the average fraction of $\pi^{0}$, s produced in pion induced showers. Similar extractions of $e / h$ for iron/scintillator sampling calorimeter was done by the CDF End Plug Upgrade group, see J.B. Liu et al., CDF Coll., CALOR97 Proceedings.

We have also compared relative energy resolution of the combined $\mathrm{PbWO}_{4}$ ECAL+HCAL system for three cases of the HCAL absorber samplings: $3 \mathrm{~cm} \mathrm{Cu}$ sampling for the first eight layers followed by $6 \mathrm{~cm} \mathrm{Cu}$ sampling, $6 \mathrm{~cm} \mathrm{Cu}$ uniform sampling and $12 \mathrm{~cm} \mathrm{Cu}$ uniform sampling. The study indicates that the energy resolution is not dominated by the sampling fluctuations in HCAL, but by the poor $e / h$ of the $\mathrm{PbWO}_{4}$ ECAL system. Therefore a factor of two change in the sampling frequency ( $3 \mathrm{~cm} / 6 \mathrm{~cm}$ vs $6 \mathrm{~cm}$ uniform sampling) of HCAL does not result in a noticeable degradation of the energy resolution of the detector. In the case of a $12 \mathrm{~cm} \mathrm{Cu}$ sampling, the degradation in energy resolution is noticeable, but did not scale with $\sqrt{t}$ ( where $t$ is the thickness of the absorber plates). This indicates that even in high pseudorapidity regions of the 
calorimeter, the sampling fluctuations do not dominate the overall resolution of the ECAL+HCAL system.

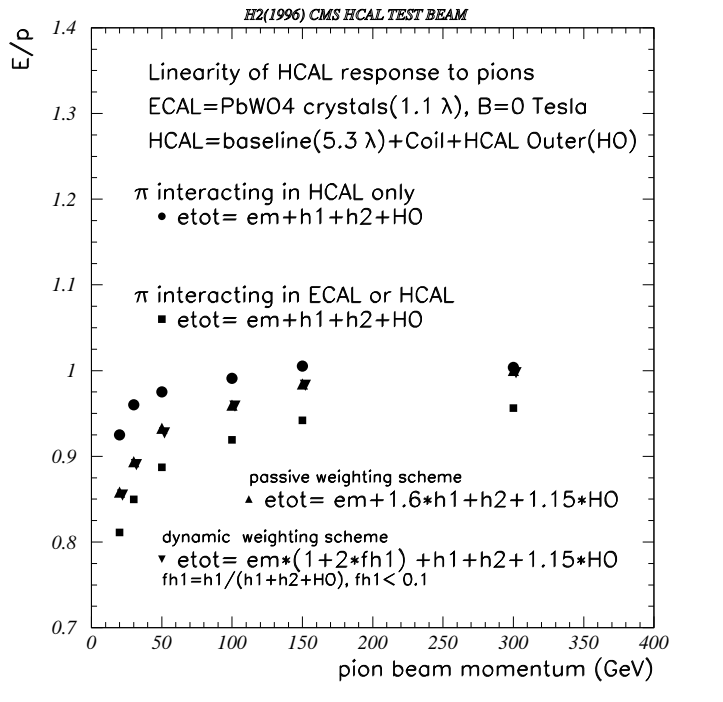

Figure 2: The linearity of the response to pions of HCAL, and the combined $\mathrm{PbWO}_{4}$ ECAL + HCAL system. Statistical error bars are smaller than symbols. The ECAL corresponds to $1.1 \lambda_{I N T}$ of lead tungstate crystals. The HCAL readout corresponds to the Baseline Inner HCAL+HCAL Outer, as defined below. The Inner $\operatorname{HCAL}\left(5.3 \lambda_{I N T}\right)$ consists of two independent readouts: H1 (following a $2 \mathrm{~cm} \mathrm{Cu}$ plate) and $\mathrm{H} 2$ (thirteen $6 \mathrm{~cm} \mathrm{Cu}$ samplings). A single readout HCAL Outer $(\mathrm{HO})$ consists of three samplings: immediately after the magnetic coil (mimicked by $18 \mathrm{~cm}$ of $\mathrm{Cu}$ ), followed by a $22 \mathrm{~cm} \mathrm{Cu}$ sampling and a $16 \mathrm{~cm} \mathrm{Cu}$ sampling. The combined coil and HO samplings corresponds to $2.5 \lambda_{I N T}$. The scintillator plates are $4 \mathrm{~mm}$ thick SCSN-81.

\section{REFERENCES}

[1] CMS Technical Proposal, CERN/LHCC 94-38, December $15,1994$.

[2] CMS HCAL Tech. Design Report, CERN/LHCC 97-31, June 20, 1997.

[3] Scintillator Tile-Fiber Calorimeters for High Energy Physics, The CDF End Plug Upgrade, Selected Articles, edited by P. de Barbaro and A. Bodek, U. of Rochester, UR-1309, October 1994.

[4] S. Kunori et al., CMS Coll., CALOR97 conference proceedings Magnetic Fields and Calorimeter Performance,

[5] V. Abramov et al., IHEP preprint 96-91. Study of Magnetic Field Influence on Hadron Calorimeter Response,

[6] R. Wigmans, NIM A265, 273-290(1988).

[7] T.A. Gabriel et al., NIM A338, 336-347(1994).

[8] J. B. Liu et al., CDF Coll., CALOR97 conference proceedings. Testbeam Results For the CDF END PLUG Hadron Calorimeter,

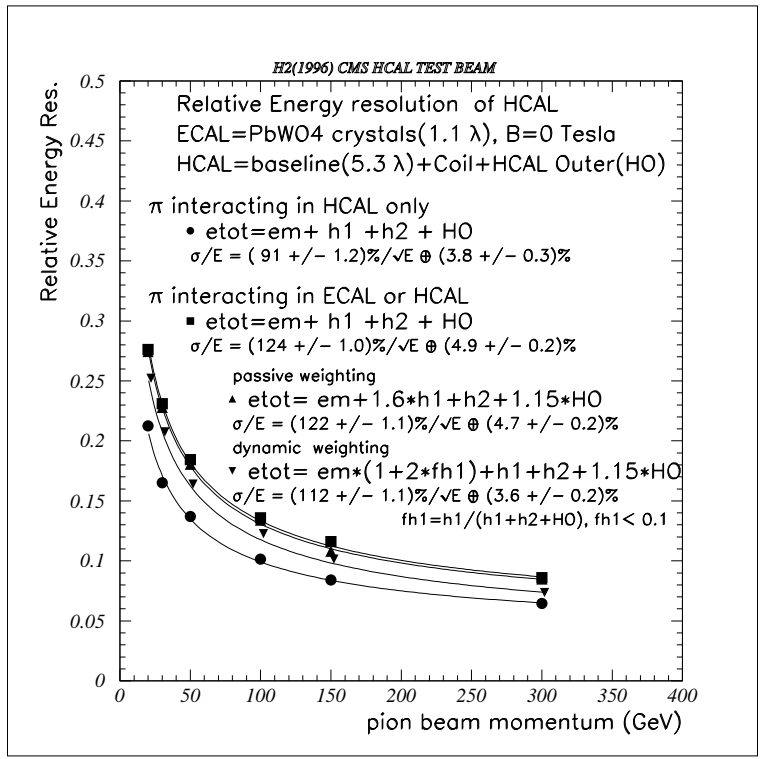

Figure 3: The relative pion energy resolution of HCAL, and the combined $\mathrm{PbWO}_{4}$ ECAL + HCAL system. Statistical error bars are smaller than symbols. The ECAL corresponds to $1.1 \lambda_{I N T}$ of lead tungstate crystals. The HCAL readout corresponds to the Baseline Inner HCAL+HCAL Outer, as defined below. The Inner $\operatorname{HCAL}\left(5.3 \lambda_{I N T}\right)$ consists of two independent readouts: H1 (following a $2 \mathrm{~cm} \mathrm{Cu}$ plate) and $\mathrm{H} 2$ (thirteen $6 \mathrm{~cm} \mathrm{Cu}$ samplings). A single readout HCAL Outer $(\mathrm{HO})$ consists of three samplings: immediately after the magnetic coil (mimicked by $18 \mathrm{~cm}$ of $\mathrm{Cu}$ ), followed by a $22 \mathrm{~cm} \mathrm{Cu}$ sampling and a $16 \mathrm{~cm} \mathrm{Cu}$ sampling. The combined coil and HO samplings corresponds to $2.5 \lambda_{I N T}$. The scintillator plates are $4 \mathrm{~mm}$ thick SCSN-81. 